\title{
A WEDGE-FLOW APPROACH TO LUBRICATION THEORY*
}

\author{
BY \\ W. E. LANGLOIS \\ International Business Machines Corporation, San Jose, California
}

\begin{abstract}
A theory of fluid-film lubrication is developed from an assumption of local wedge-flow, rather than local parallel-channel flow. This leads to a generalization of the Reynolds lubrication equation governing the pressure. A refinement of lubrication theory does not necessarily follow: unlike the parallel-channel case, the stress field may differ significantly from an isotropic pressure, so that imposing ambient conditions on the bearing periphery does not always yield boundary conditions for the pressure equation. If the bearing slope differs appreciably from zero only in the film interior, consistent boundary conditions are once more available.
\end{abstract}

1. Introduction. In traditional approaches to the theory of fluid-film lubrication. it is assumed, explicitly or implicitly, that the local velocity profile is parabolic. That is to say, at a point where the lubricating film has thickness $h$, the velocity components and pressure gradients assume values which could obtain if the bearing surfaces were replaced by a pair of parallel plates with spacing $h$. Since, in general, the film thickness varies from point to point, so do the velocity profile and pressure gradient. The hydrodynamical continuity equation imposes a constraint upon the point-to-point variation of the velocity profile which, in turn, leads to a second order differential equation for the pressure. For steady, two dimensional, incompressible flow, the constraint imposed by the continuity equation is equivalent to a statement that the same volume of fluid must flow through each cross-section of the bearing per unit time. The differential equation for the pressure is subjected to the boundary condition that the pressure be ambient at the bearing periphery. The use of this boundary condition entails two approximations, both of which are consistent with the assumptions used in deriving the differential equations: the contribution of the strain-rate term to the stress tensor is negligible compared with the contribution of the pressure term; the adjustment to ambient conditions at the bearing periphery takes place in a region whose width is small compared with the lateral dimensions of the bearing. For the details of the mathematical foundations underlying the steady-state theory of incompressible lubrication, see [1], Chap. 9.

In this paper, we present a somewhat more general approach to the two-dimensional theory of steady-state lubrication by a film of incompressible fluid. We assume that wedge flow, rather than parallel-channel flow, obtains locally. This leads to Reynolds' lubrication equation in a generalized form, which allows for the possibility that the slope of the bearing surface may not be everywhere small compared with unity (the driving surface is assumed flat).

The problem of creeping viscous flow in a wedge-shaped region was treated by Milne [2], who permitted arbitrary motion of the bearing surface in addition to the sliding motion of the driving surface. With the methods set out in the present paper, Milne's

*Received March 23, 1964; revised manuscript received May 25, 1964. This work was supported in part by the United States Office of Naval Research under Contract Nonr 3448(00), Task NR 061-120. 
solution could presumably be extended to bearings which can be approximated locally by a wedge. The analysis would, however, be exceedingly complicated.

Chapter 7 of [1] includes a local-wedge-flow approximation method for pressure flow of a highly viscous fluid through a channel of varying gap. The treatment here is completely analogous, but the problem is more involved.

2. Creeping flow in a wedge. Consider first the problem of creeping viscous flow in a wedge-shaped region (Fig. 1). If the walls of the wedge are stationary, the flow

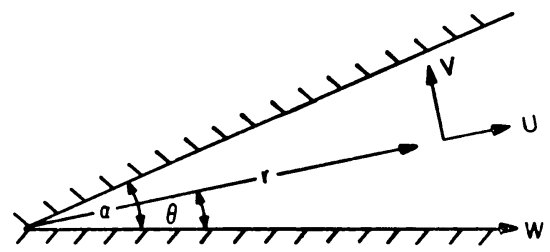

FIG. 1. Wedge flow

generated by a line source lying along the vertex of the wedge is purely radial and has been widely studied. For application to lubrication theory, however, we require that one surface of the wedge be in steady motion parallel to itself. We denote its velocity by $W$ and the strength of the source by $Q$.

For the polar coordinate system shown in Fig. 1, the equations of two-dimensional creeping flow of a fluid with viscosity $\mu$ are

$\frac{\partial}{\partial r}(r U)+\frac{\partial V}{\partial \theta}=0, \frac{\partial p}{\partial r}=\mu\left(\nabla^{2} U-\frac{U}{r^{2}}-\frac{2}{r^{2}} \frac{\partial V}{\partial \theta}\right), \quad \frac{\partial p}{\partial \theta}=\mu r\left(\nabla^{2} V+\frac{2}{r^{2}} \frac{\partial U}{\partial \theta}-\frac{V}{r^{2}}\right)$,

where $p$ denotes the pressure, $U$ and $V$ denote, respectively, the radial and azimuthal components of velocity.

The no-slip condition at the walls requires that

$$
U=W, \quad V=0 \text { at } \theta=0 ; \quad U=V=0 \text { at } \theta=\alpha .
$$

Finally, we have the volume flow condition

$$
\int_{0}^{\alpha} r U d \theta=Q
$$

which must hold for each value of $r$.

Through direct substitution, we can verify that the above equations and boundary conditions are satisfied by setting

$$
\begin{aligned}
& U=\left(W / E_{2}\right)\left[\left(E_{2}-E_{1} \theta\right) \cos \theta+\left(\theta \tan ^{2} \alpha-E_{1}\right) \sin \theta\right] \\
& +(Q / \mathrm{r})[\cos (2 \theta-\alpha)-\cos \alpha] /[\sin \alpha-\alpha \cos \alpha], \\
& V=\left(W / E_{2}\right)\left[\left(\tan ^{2} \alpha\right) \theta \cos \theta+\left(E_{1} \theta-\alpha^{2} \sec ^{2} \alpha\right) \sin \theta\right], \\
& p=C-\left(2 \mu W / E_{2} r\right)\left[\left(\tan ^{2} \alpha\right) \cos \theta+E_{1} \sin \theta\right] \\
& +\left(2 \mu Q / r^{2}\right)[\cos (2 \theta-\alpha)] /[\sin \alpha-\alpha \cos \alpha],
\end{aligned}
$$

where

$$
E_{n}=\alpha^{n} \sec ^{2} \alpha-\tan ^{n} \alpha, \quad(n=1,2)
$$

and $C$ is a constant of integration (the pressure infinitely far from the source). 
3. The local wedge-flow approximation. Assume now that the lubricating film is contained between a flat driving surface and a curved bearing surface. We assume that the bearing surface is curved only in the direction of motion and that the bearing is infinitely long in the direction transverse to the motion. Moreover, we assume that the curvature of the bearing surface is gentle enough so that the bearing can be approximated locally by a wedge, as illustrated in Fig. 2.

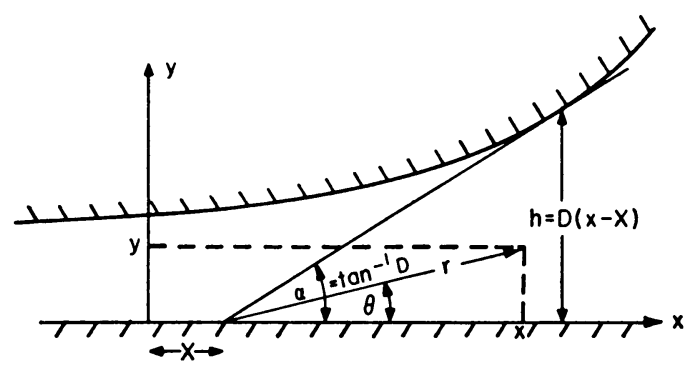

FIG. 2. Bearing approximated locally by a wedge

The slope $D$ is not assumed small compared with unity. However, the local Reynolds number is proportional to $h(x)$; hence, the discussion to follow involves the tacit assumption that moderate to large values of $D(x)$ do not extend over a wide enough range to vitiate the creeping flow assumption.

We approximate the $r$ and $\theta$ components of velocity at the point $(x, y)$ by the expressions (2.4). It is erroneous to approximate $p$ by (2.5), for the integrated value of the pressure will, in general, receive contributions from portions of the film where the slope of the bearing surface is quite different from $D$. Instead, we assume that the local components of the pressure gradient are approximated by the derivatives of (2.5):

$\partial p / \partial r=\left(2 \mu W / E_{2} r^{2}\right)\left[\left(\tan ^{2} \alpha\right) \cos \theta+E_{1} \sin \theta\right]$

$$
-\left(4 \mu Q / r^{3}\right)[\cos (2 \theta-\alpha)] /[\sin \alpha-\alpha \cos \alpha],
$$

$\partial p / \partial \theta=\left(2 \mu W / E_{2} r\right)\left[\left(\tan ^{2} \alpha\right) \sin \theta-E_{1} \cos \theta\right]$

$$
-\left(4 \mu Q / r^{2}\right)[\sin (2 \theta-\alpha)] /[\sin \alpha-\alpha \cos \alpha] .
$$

We now replace the polar coordinate system by the Cartesian system $(x, y)$ as illustrated in Fig. 2. Thus

$$
x=X+r \cos \theta, \quad y=r \sin \theta .
$$

If we denote the components of velocity in the increasing $x$ and $y$ directions by $u$ and $v$ respectively, we have

$$
\begin{aligned}
& u=U \cos \theta-V \sin \theta=\frac{(x-X) U-y V}{\left[(x-X)^{2}+y^{2}\right]^{1 / 2}}, \\
& v=V \cos \theta+U \sin \theta=\frac{(x-X) V+y U}{\left[(x-X)^{2}+y^{2}\right]^{1 / 2}} .
\end{aligned}
$$

The somewhat ficticious length $X$ can be removed from these transformations by noting that

$$
h=D(x-X) .
$$


Substituting equations (2.5) into (3.3) then gives us

$$
\begin{aligned}
& u=W\left(1+\frac{D^{3} y-E_{1} h}{h^{2}+D^{2} y^{2}} \frac{D y}{E_{2}}-\frac{E_{1}}{E_{2}} \arctan \frac{D y}{h}\right)+\frac{2 Q D^{3}}{D-\arctan D} \frac{h y(h-y)}{\left(h^{2}+D^{2} y^{2}\right)^{2}}, \\
& v=\frac{W D^{2}}{E_{2}}\left(\arctan \frac{D y}{h}-\frac{E_{1} y^{2}+D h y}{h^{2}+D^{2} y^{2}}\right)+\frac{2 Q D^{4}}{D-\arctan D} \frac{y^{2}(h-y)}{\left(h^{2}+D^{2} y^{2}\right)^{2}} .
\end{aligned}
$$

The quantities $E_{1}, E_{2}$ which appear in these expressions are defined by (2.6). In terms of $D$ rather than $\alpha$,

$$
E_{n}=\left(1+D^{2}\right)(\arctan D)^{n}-D^{n} \quad(n=1,2) .
$$

For the components of the pressure gradient, we have

$$
\begin{aligned}
& \frac{\partial p}{\partial x}=\frac{\partial p}{\partial r} \frac{\partial r}{\partial x}+\frac{\partial p}{\partial \theta} \frac{\partial \theta}{\partial x}=\frac{h}{\left(h^{2}+D^{2} y^{2}\right)^{1 / 2}} \frac{\partial p}{\partial r}-\frac{D^{2} y}{h^{2}+D^{2} y^{2}} \frac{\partial p}{\partial \theta} \\
& \frac{\partial p}{\partial y}=\frac{\partial p}{\partial r} \frac{\partial r}{\partial y}+\frac{\partial p}{\partial \theta} \frac{\partial \theta}{\partial y}=\frac{D y}{\left(h^{2}+D^{2} y^{2}\right)^{1 / 2}} \frac{\partial p}{\partial r}+\frac{D h}{h^{2}+D^{2} y^{2}} \frac{\partial p}{\partial \theta}
\end{aligned}
$$

With Eqs." (3.1), (3.2), and (3.4), we then obtain

$$
\begin{aligned}
& \frac{\partial p}{\partial x}=\frac{2 \mu W D^{3}}{E_{2}} \frac{D h^{2}+2 E_{1} h y-D^{3} y^{2}}{\left(h^{2}+D^{2} y^{2}\right)^{2}}-\frac{4 \mu Q D^{3}}{D-\arctan D} \frac{h^{3}+3 D h^{2} y-3 D^{2} h y^{2}-D^{4} y^{3}}{\left(h^{2}+D^{2} y^{2}\right)^{3}}, \\
& \frac{\partial p}{\partial y}=-\frac{2 \mu W D^{2}}{E_{2}} \frac{E_{1} h^{2}-2 D^{3} h y-D^{2} E_{1} y^{2}}{\left(h^{2}+D^{2} y^{2}\right)^{2}}+\frac{4 \mu Q D^{4}}{D-\arctan D} \frac{h^{3}-3 h^{2} y-3 D^{2} h y^{2}+D^{2} y^{3}}{\left(h^{2}+D^{2} y^{2}\right)^{3}} .
\end{aligned}
$$

In Cartesian coordinates, the equations of creeping flow are

$$
\partial p / \partial x=\mu \nabla^{2} u, \quad \partial p / \partial y=\mu \nabla^{2} v .
$$

We expect that the expressions (3.5) and (3.8) will provide an approximate solution to this system, provided that the rate of change of $D$ is sufficiently slow. This condition requires codification, for $D^{\prime}(x)$ is a dimensional quantity, and it is meaningless to speak of its being "small." However, $h(x) D^{\prime}(x)$ is dimensionless and does, in fact, provide a measure of the validity of (3.5) and (3.8). Geometrically, if the curvature of the bearing surface is large, the film can be approximated locally by a wedge if and only if the gap is correspondingly small, i.e., if and only if

$$
h(x) D^{\prime}(x) \ll 1 .
$$

Since second derivatives appear in equations (3.9), we need also

$$
h^{2} D^{\prime \prime} \ll 1,
$$

which says, in effect, that sharp changes in curvature do not occur. If (3.10) and (3.11) are satisfied, it is not difficult to verify by direct substitution that (3.5) and (3.8) satisfy the flow equations (3.9).

If (3.10) is obeyed, so that terms in $h(x) D^{\prime}(x)$ can be neglected, we see that, with $\partial p / \partial x$ and $\partial p / \partial y$ given by (3.8),

$$
d p=\frac{\partial p}{\partial x} d x+\frac{\partial p}{\partial y} d y
$$


is an exact differential. Therefore,

$$
p=\int_{C, 0}^{x, \nu}\left(\frac{\partial p}{\partial x} d x+\frac{\partial p}{\partial y} d y\right)
$$

where $C$ is a constant. If we choose the path of integration as illustrated in Figure 3, we obtain

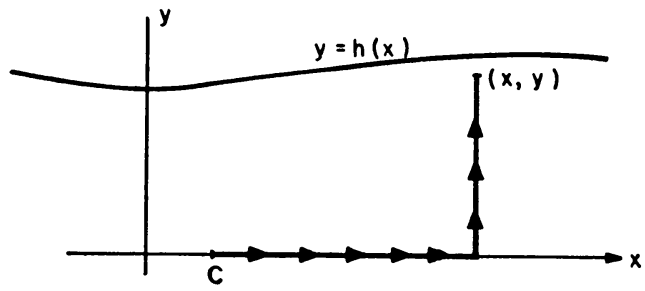

FIG. 3. Line integral for the pressure

$$
p(x, y)=p_{0}(x)-\frac{2 \mu W D^{2}}{E_{2} h} I\left(\frac{y}{h}\right)+\frac{4 \mu Q D^{4}}{(D-\arctan D) h^{2}} J\left(\frac{y}{h}\right)
$$

where

$$
\begin{aligned}
p_{0}(x) & =2 \mu \int_{C}^{x}\left[\frac{W D^{4}}{E_{2} h^{2}}-\frac{2 Q D^{3}}{(D-\arctan D) h^{3}}\right] d x, \\
I(z) & =\int_{0}^{z} \frac{E_{1}\left(1-D^{2} z^{2}\right)-2 D^{3} z}{\left(1+D^{2} z^{2}\right)^{2}} d z=\frac{E_{1} z-D^{3} z^{2}}{1+D^{2} z^{2}} \\
J(z) & =\int_{0}^{z} \frac{1-3 z-3 D^{2} z^{2}+D^{2} z^{3}}{\left(1+D^{2} z^{2}\right)^{3}} d z=\frac{2 z-3 z^{2}-D^{2} z^{4}}{2\left(1+D^{2} z^{2}\right)^{2}} .
\end{aligned}
$$

4. Pressure variation along the driving surface. Since $I(z)$ and $J(z)$ both vanish at $z=0$, the pressure at a generic point $x$ on the driving surface is $p_{0}(x)$. By setting $y=0$ in the first of equations (3.8), or by differentiating (3.15), we see that $p_{0}$ obeys the ordinary differential equation

$$
\frac{h^{3}}{2 \mu} \frac{d p_{0}}{d x}=\frac{W D^{4} h}{E_{2}}-\frac{2 Q D^{3}}{D-\arctan D} .
$$

Equation (3.14) can then be rewritten as follows

$$
p(x, y)=p_{0}(x)-D h p_{0}^{\prime}(x) J\left(\frac{y}{h}\right)+\frac{2 \mu W D^{2}}{E_{2} h} K\left(\frac{y}{h}\right),
$$

where

$$
K(z)=D^{3} J(z)-K(z)=\frac{z}{2\left(1+D^{2} z^{2}\right)^{2}}\left[D^{3}\left(2-z-D^{2} z^{3}\right)-2 E_{1}\left(1+D^{2} z^{2}\right)\right] .
$$

Let us consider briefly the special case of $D$ small compared with unity. Since

$$
\arctan D=D-D^{3} / 3+D^{5} / 5+O\left(D^{7}\right) \text {, }
$$

Eq. (3.6) yields

$$
E_{2}=\left(D^{4} / 3\right)\left[1-(7 / 15) D^{2}+O\left(D^{4}\right)\right]
$$


Consequently, with neglect only of terms of the second degree or higher in $D$, Eq. (4.1) reduces to

$$
\frac{h^{3}}{6 \mu} \frac{d p_{0}}{d x}=W h-2 Q,
$$

which is easily recognized as a first integral of the classical Reynolds lubrication equation

$$
\frac{d}{d x}\left(h^{3} \frac{d p_{0}}{d x}\right)=6 \mu W \frac{d h}{d x} .
$$

Returning to the case in which $D$ is a finite function of $x$, we observe that (4.1) can be considered as a first integral of a second order differential equation, which might be termed a generalized Reynolds equation. Differentiating (4.1) yields

$$
\frac{d}{d x}\left(\frac{h^{3}}{2 \mu} \frac{d p_{0}}{d x}\right)=\frac{W D^{5}}{E_{2}}+h \frac{d D}{d x} \frac{d}{d D}\left(\frac{W D^{4}}{E_{2}}-\frac{2 Q D^{3}}{D-\arctan D}\right) .
$$

At first glance, it would appear that the second term on the right side of (4.8) could be dropped, for it involves $h(x) D^{\prime}(x)$, which we have assumed negligible. However, we cannot ignore the cumulative contribution of this term when (4.8) is integrated. Even though $D$ is a slowly varying function of $x$, it undergoes large-scale changes over the breadth of the bearing; typically, $D(x)$ is negative in the inlet region, positive in the outlet region. The appropriate second order differential equation is obtained by carrying out the differentiation indicated on the right side of (4.8) and using (4.1) to eliminate $Q$ :

$$
\frac{d}{d x}\left(\frac{h^{3}}{2 \mu} \frac{d p_{0}}{d x}\right)=W \frac{d}{d x}\left(\frac{D^{4} h}{E_{2}}\right)+\frac{1}{D}\left(\frac{2 D^{3}-3 E_{1}}{D^{3}-E_{1}}\right)\left(\frac{h^{3}}{2 \mu} \frac{d p_{0}}{d x}-\frac{W D^{4} h}{E_{2}}\right) \frac{d D}{d x}
$$

5. Stress components and boundary conditions. In Newtonian viscous flow, the stress matrix $\mathbf{T}$ is related to the flow variables according to

$$
\mathbf{T}=-p \mathbf{I}+2 \mu \mathrm{E},
$$

where $\mathbf{I}$ is the unit matrix and $\mathbf{E}$ is the strain-rate matrix, defined by

$$
\mathbf{E}=\left\|\begin{array}{cc}
\frac{\partial u}{\partial x} & \frac{1}{2}\left(\frac{\partial u}{\partial y}+\frac{\partial v}{\partial x}\right) \\
\frac{1}{2}\left(\frac{\partial u}{\partial y}+\frac{\partial v}{\partial x}\right) & \frac{\partial v}{\partial y}
\end{array}\right\| .
$$

With the velocity components given by (3.5),

$$
\mathbf{E}=\frac{W D}{E_{2} h} \mathbf{E}_{\diamond}\left(\frac{y}{h} ; D\right)+\frac{2 Q D^{3}}{(D-\arctan D) h^{2}} \mathbf{E}_{\odot}\left(\frac{y}{h} ; D\right),
$$

where the matrix functions $E_{0}$ and $\mathbf{E}_{Q}$ are defined by

$$
\mathrm{E}_{\diamond}(Z ; D)=\frac{E_{1}-D^{3} Z}{\left(1+D^{2} Z^{2}\right)^{2}}\left\|\begin{array}{cc}
2 D Z & D^{2} Z^{2}-1 \\
D^{2} Z^{2}-1 & -2 D Z
\end{array}\right\|,
$$

$\mathbf{E}_{\odot}(Z ; D)=\frac{1}{2\left(1+D^{2} Z^{2}\right)^{3}}$.

$$
\text { . }\left\|\begin{array}{cc}
-2 D Z\left(2-3 Z-2 D^{2} Z^{2}+D^{2} Z^{3}\right) & \left(1-2 Z-6 D^{2} Z^{2}+6 D^{2} Z^{3}+D^{4} Z^{4}\right) \\
\left(1-2 Z-6 D^{2} Z^{2}+6 D^{2} Z^{3}+D^{4} Z^{4}\right) & 2 D Z\left(2-3 Z-2 D^{2} Z^{2}+D^{2} Z^{3}\right)
\end{array}\right\|
$$


Using (4.1) to eliminate $Q$ from (5.3), we obtain

$$
\mathbf{E}=\frac{W D}{E_{2} h}\left[\mathbf{E}_{\diamond}\left(\frac{y}{h} ; D\right)+D^{3} \mathbf{E}_{\odot}\left(\frac{y}{h} ; D\right)\right]-\frac{h}{2 \mu} \frac{d p_{0}}{d x} \mathbf{E}_{\odot}\left(\frac{y}{h} ; D\right) .
$$

The stresses in the fluid can now be obtained by substituting (4.2) and (5.6) into (5.1). Thus,

$$
\mathbf{T}(x, y ; D)=-p_{0}(x) \mathbf{I}+h p_{0}^{\prime}(x) \mathbf{F}\left(\frac{y}{h} ; D\right)+(2 \mu W / h) \mathbf{G}\left(\frac{y}{h} ; D\right),
$$

where

$$
\begin{gathered}
\mathbf{F}(Z ; D)=D J(Z) \mathbf{I}-\mathbf{E}_{o}(Z), \\
\mathbf{G}(Z ; D)=\left(D / E_{2}\right)\left[\mathbf{E}_{s}(Z)-D K(Z) \mathbf{I}+D^{3} \mathrm{E}_{o}(Z)\right] .
\end{gathered}
$$

In general, the lubrication region constitutes only part of the flow field. The full hydrodynamical problem should take into account the entire geometry of the region occupied by the lubricant. In classical lubrication theory, however, one usually assumes that the bearing terminates at more-or-less well defined end points, beyond which ambient conditions obtain. Thus, if $p_{a}$ denotes the ambient pressure, it is assumed that

$$
\mathbf{T}=-p_{a} \mathbf{I} \text { at } x=x_{0}, x_{0}+B,
$$

where $x_{0}$ and $x_{0}+B$ are the ends of the bearing. Moreover, it is established [1] that $p_{0}(x)$ is of order $(h / B)^{-2}$ for small $(h / B)$, so that $\mathbf{T}(x, y ; 0)$ is adequately represented by $-p_{0}(x) \mathbf{I}$.

In the more general case considered here, there is no guarantee that boundary conditions on $p_{0}(x)$ adequately describe the coupling of the lubricating film with the external environment. There are three difficulties: it may not be possible to specify precise values of $x_{0}$ and $x_{0}+B$ at which ambient conditions are adequately approximated; the deviatoric part of $\mathbf{T}(x, y ; D)$ may be of the same order as the pressure; the $y$-variation of $\mathbf{T}(x, y ; D)$ may be of the same order as $p_{0}(x)$.

If the bearing surface slope differs appreciably from zero only in the interior of the film, as illustrated in Fig. 4, there is no problem. At the ends of the bearing, the stress

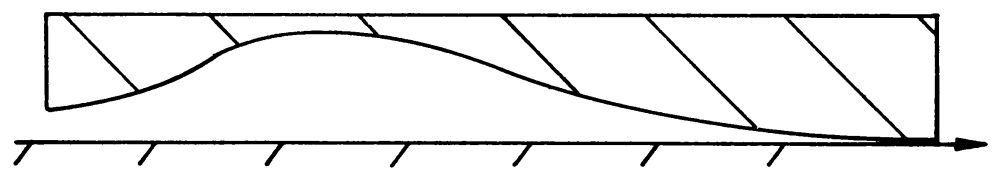

FIG. 4.

tensor is adequately represented by $-p_{0}(x) \mathbf{I}$, which can be matched to the ambient value $-p_{a} \mathbf{I}$.

When the region of finite slope occurs near an end of the bearing, it can easily happen that there is no meaningful way to specify boundary conditions on $p_{0}(x)$. Each case must be considered individually. Basically, a double limit problem is involved. To proceed beyond classical theory, we must retain terms in $D(x)$. However, to obtain boundary conditions on $p_{0}(x)$ we need

$$
h(x) / B^{*} \ll 1,
$$


where $B^{*}$ is the length over which $D(x)$ differs appreciably from zero. If $D(x)$ has the same sign throughout the region $\left(x_{0}, x_{0}+B^{*}\right)$, then

$$
h\left(x_{0}+B^{*}\right)=\int_{x_{0}}^{x_{0}+B^{*}} D(x) d x=B^{*} D_{0},
$$

with the mean value theorem for integrals assuring that $D_{0}=D_{0}(x)$ for some $x$ in $\left(x_{0}, x_{0}+B^{*}\right)$. Hence we cannot simultaneously have $D(x)$ large and $h(x) / B^{*}$ everywhere small.

When the region of finite slope occurs near an end of the bearing, but $D(x)$ changes sign within this region-as appears to be the case near the trailing edge of a foil bearing [3]- we may be able to retain terms resulting from the wedge flow and yet obtain boundary conditions. It depends upon the relative magnitudes of $D(x)$ and $h(x) / B^{*}$ for the problem under consideration.

\section{REFERENCES}

1. W. E. Langlois: Slow viscous flow;Macmillian, 1964

2. A. A. Milne: A contribution to the theory of hydrodynamic lubrication: A solution in terms of the stream function for a wedge-shaped oil film; Wear 1, (1957) 32

3. W. A. Gross: Gas film lubrication; Wiley, 1962; pages 138-141 